\title{
The causes of obvious jaundice in South West Wales: perceptions versus reality
}

\author{
M W Whitehead, I Hainsworth, J G C Kingham
}

\begin{abstract}
Aims-(1) A prospective analysis of clinically obvious jaundice (bilirubin $>120$ $\mu \mathrm{mol} / 1)$ in South Wales to determine accuracy of diagnosis, referral pattern, treatment, and outcome. (2) To compare British gastroenterologists' and local general practitioners' perceptions of common causes of jaundice with our study findings.

Methods-Over a seven month period all patients with bilirubin $>120 \mu \mathrm{mol} / 1$ (excluding neonates with physiological jaundice) were identified by a biochemistry laboratory serving three general hospitals and the community. Clinical data were recorded prospectively. Sixty nine consultant gastroenterologists and 67 local general practitioners (GPs) were asked to cite the commonest causes of bilirubin $>120 \mu \mathrm{mol} / 1$ in their experience.

Results-A total of 121 patients were identified of whom 95 were admitted to hospital because of jaundice, 22 developed jaundice while in hospital, and four remained in the community. Causes of jaundice were: malignancy 42, sepsis/ shock 27 , cirrhosis 25 , gall stones 16 , drugs 7 , autoimmune hepatitis 2, and viral hepatitis 2. One in five was wrongly diagnosed, often as viral hepatitis. Although $30 \%$ were under surgical care only $4 \%$ required surgery. Overall mortality was high $(31 \%)$ and greatest in sepsis/ shock $(51 \%)$. Gastroenterologists and GPs both perceived malignancy and gall stones to be the commonest causes of marked jaundice followed by viral hepatitis and cirrhosis; sepsis/shock was hardly mentioned.
\end{abstract}

Conclusions-There are important discrepancies between gastroenterologists' and GPs' perceptions of likely causes of jaundice and the actual causes we have shown. In particular, sepsis/shock is common in hospital practice but is overlooked whereas viral hepatitis is rare but perceived as common and overdiagnosed. Gall stones usually cause mild jaundice with bilirubin levels less than $120 \mu \mathrm{mol} / 1$. Many patients are referred to surgical services for historical reasons yet rarely require surgery and are usually treated by physicians or endoscopists.

(Gut 2001;48:409-413)

Keywords: jaundice; bilirubin; sepsis; hepatitis; gall stones; questionnaire
Jaundice is a common problem in medical and surgical gastroenterological practice. Its cause can often be correctly anticipated from the clinical circumstances but investigations are usually required for confirmation. It is much less common in primary care and in hospital practice outside gastroenterology and hepatobiliary surgery. It has been our perception that jaundice is often wrongly attributed when it is first seen in these less specialised settings. Part of the problem is that clinicians, although aware of the many possible causes of jaundice, are uncertain as to which are common and which are rare in a particular community.

We therefore performed a prospective study of all causes of clinically obvious jaundice in a well defined and stable population in South Wales. The objects were to ascertain the accuracy of primary diagnosis, referral patterns from primary care to hospital departments, causes, treatments, and outcomes. A bilirubin level greater than $120 \mu \mathrm{mol} / 1$ was chosen to define obvious jaundice as we believed that jaundice of this degree would be unlikely to escape the attention of the patient or attending doctor. We anticipated that in most cases a blood sample would be taken to confirm the clinical diagnosis. All cases of bilirubin $>120$ $\mu \mathrm{mol} / 1$ were identified by the central biochemical laboratory serving this part of South Wales and so it was envisaged that our survey would encompass the majority of patients with obvious jaundice in this population.

\section{Methods}

All serum samples with bilirubin $>120 \mu \mathrm{mol} / 1$ (normal up to 25) measured by a central biochemistry department were identified. This laboratory serves three district general hospitals and the community of West Glamorgan with a population of approximately 369000 . The survey was undertaken between August 1997 and February 1998. The population served is $51 \%$ female and is predominantly of European ethnic origin (99\%). ONS values for 1996-1997 show a $0.1 \%$ population growth with little emigration or immigration.

Bilirubin was measured with the Bayer Dax System using the reaction with diazotised sulphanilic acid with a surfactant as an accelerator. ${ }^{1}$

The biochemistry department informed the clinicians involved in the study of the names and whereabouts of the patients identified on a daily basis. If the patient was in hospital, the

Abbreviations used in this paper: GP, general practitioner; BSG, British Society of Gastroenterology; AST, aspartate aminotransferase; ITU, intensive therapy unit; $\mathrm{CBD}$, common bile duct.
Accepted for publication 17 October 2000 
Table 1 Location of patients by hospital specialty (medicine includes care of the elderly and renal medicine)

\begin{tabular}{lcl}
\hline & Initial & Final \\
\hline Gastroenterology & $34(29 \%)$ & $40(34 \%)$ \\
General medicine & $35(30 \%)$ & $27(23 \%)$ \\
Surgery & $35(30 \%)$ & $25(21 \%)$ \\
ITU & 0 & $10(9 \%)$ \\
Haemato-oncology & $10(9 \%)$ & $12(10 \%)$ \\
Paediatrics & $3(3 \%)$ & $3(3 \%)$ \\
\hline
\end{tabular}

clinical record was examined and the age, sex, hospital speciality, relevant medical and drug history, and results of investigations were obtained. Provisional and final diagnoses, and clinical outcome were determined by review of the notes at the end of the hospital admission. If the patient was in the community the general practitioner (GP) was contacted by telephone and the case history discussed. All patients other than neonates with physiological jaundice were included in the study.

A postal survey of a representative sample of 75 senior British Society of Gastroenterology (BSG) members and 75 local GPs was undertaken. Sixty nine BSG members and 67 GPs responded. The BSG members had generally held consultant positions for 10-20 years and were distributed around the UK in district hospitals, teaching hospitals, and liver units. Each respondent was asked to nominate the three commonest causes of bilirubin $>120$ $\mu \mathrm{mol} / 1$ in his/her experience, if possible ranked in order. The choices were awarded 3,2 , or 1 points if ranked, or 2 each if unranked. The final score for any given diagnosis was given by summation of points divided by the number of respondents. Thus the maximum achievable score for a diagnosis was 3 points.

Approval for the study was given by the local ethics committee.

\section{Results}

A total of 309 patients with bilirubin levels greater than $120 \mu \mathrm{mol} / 1$ were identified: 188 were neonates with physiological jaundice and were not considered further. The remaining 121 patients formed the basis of the study. There were 72 males and median age was 66 years (range 1-93.)

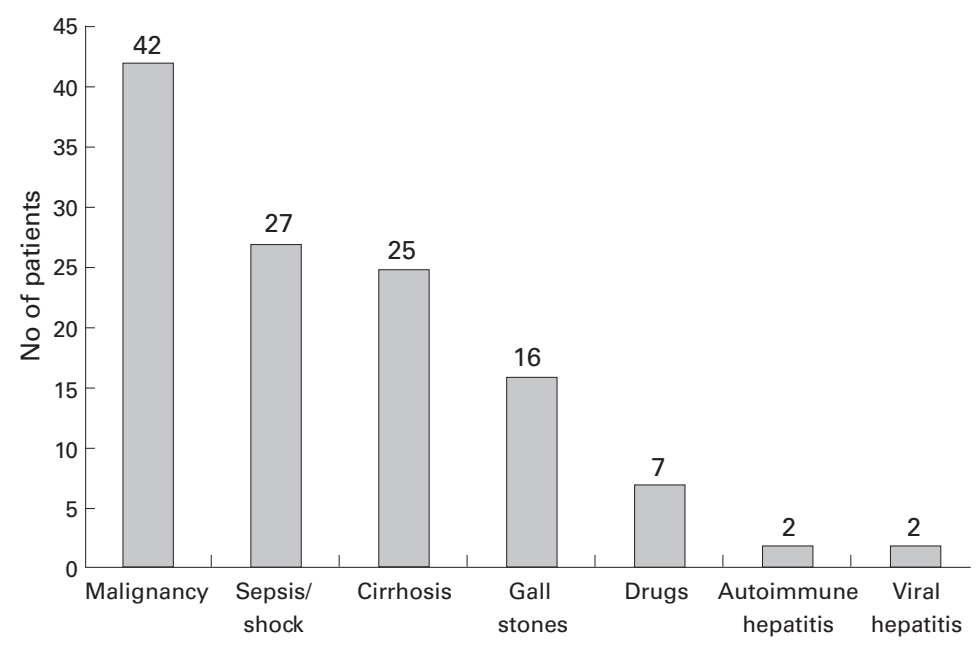

Figure 1 Causes of jaundice in the 121 patients identified.
Ninety five patients were admitted to hospital because of jaundice whereas 22 developed jaundice while in hospital for other reasons. Only four patients remained in primary care and did not require admission to hospital.

The location of patients at the time of hospital admission is shown in table 1. Thirty four $(29 \%)$ were under the care of gastroenterologists and $35(30 \%)$ under other physicians. Thirty five patients $(30 \%)$ were under the care of general surgeons, $10(9 \%)$ patients under oncologists, and three (3\%) patients under paediatricians. Following admission some patients moved from one speciality to another, as shown in table 1 . This was mainly accounted for by patients moving from general medicine and surgery to gastroenterology or the intensive therapy unit (ITU).

The causes of jaundice in the 121 patients are shown in fig 1 . The commonest cause was cancer (42 patients $(35 \%)$ ), with pancreatobiliary malignancy topping the list: 26 patients had carcinoma of the pancreas and eight had cholangiocarcinoma. In addition, there were eight patients with disseminated cancer involving the liver or compressing the biliary tree.

Surprisingly, the second commonest cause was sepsis/shock in 27 patients $(22 \%)$, of whom 11 were found in the ITU, surgical, and oncology wards. Sepsis and shock were diagnosed clinically on the basis of temperature, pulse, blood pressure, cardiorespiratory and renal function, oxygen delivery, and microbiological results. The underlying aetiology was variable; postoperative complications with multiorgan failure or shock in 13, sepsis in 13, and heart failure in one.

Thirty six patients (30\%) had hepatocellular disease of which the most frequent was alcoholic cirrhosis and/or hepatitis (25). Seven patients had jaundice caused by drugs, namely paracetamol (two), L-dopa, methyl-dopa, simvastatin, flucloxacillin, and ofloxacin. Two patients had autoimmune hepatitis (first presentation in both) and two acute viral hepatitis (hepatitis A one, Epstein-Barr virus one).

In only 16 patients $(13 \%)$ was the jaundice caused by common bile duct (CBD) stones which initially appeared anomalous. Our computerised endoscopic records for the three hospitals during the defined study period showed CBD stones in 61 of 207 cases undergoing ERCP. In these 61 patients, bilirubin levels were generally elevated but in only 16 cases was the peak value greater than $120 \mu \mathrm{mol} / 1$.

The diagnostic profile of patients whose jaundice developed while in hospital was very different from that of those admitted because of jaundice. Of the 22 patients in the former group, jaundice was related to sepsis/shock in 20 , one was drug related, and one had extensive liver metastases.

Thirty eight patients (32\%) died while in hospital. The highest mortality was in those with sepsis/shock (53\%). Mortality was also high in malignancy and cirrhosis $(24 \%$ and $23 \%$ ). Only two of 15 patients with gall stone related jaundice died and there were no deaths among those with drug jaundice or hepatitis. 


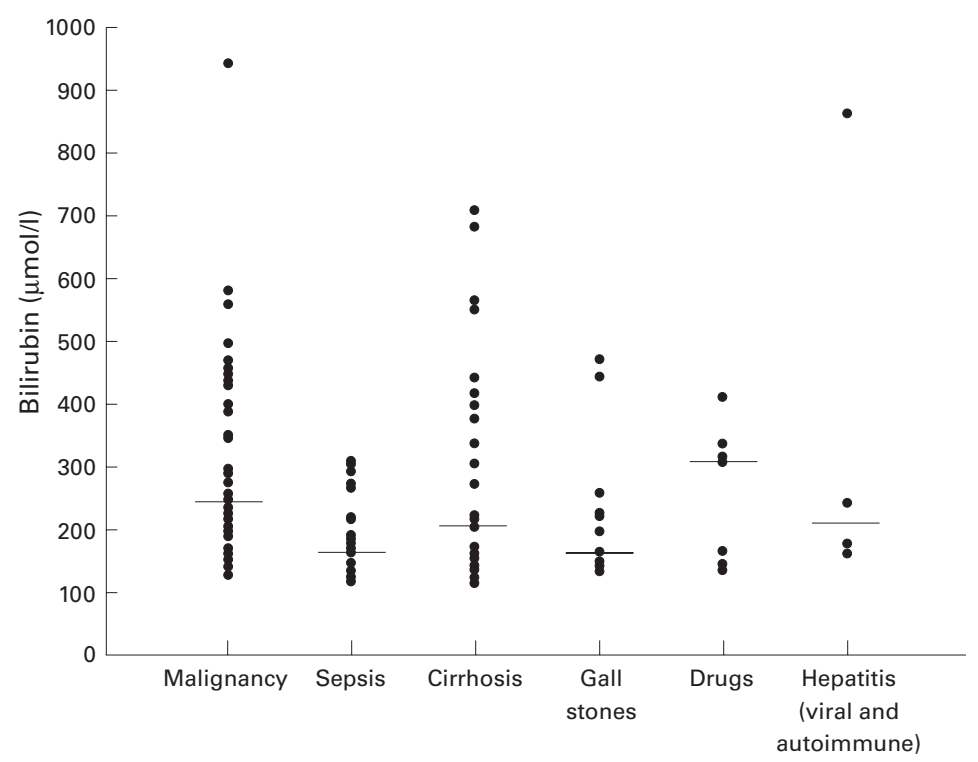

Figure 2 Peak bilirubin levels for each diagnostic group (with medians).

None of the four patients cared for in the community died.

Forty five patients (37\%) had endoscopic treatment for their jaundice; 10 had stone extraction and 35 had biliary stenting. Thirty four patients $(28 \%)$ improved on conservative treatment and were discharged without endoscopic or surgical intervention. Only five patients $(4 \%)$ had an operation (bypass for malignant obstruction, three; interval cholecystectomy, two). Two further patients with malignancy were considered for surgery but deemed unsuitable.

Median peak bilirubin levels were highest in patients with drug jaundice $(318 \mu \mathrm{mol} / \mathrm{l})$ and lowest in gall stone jaundice $(154 \mu \mathrm{mol} / \mathrm{l})$ and jaundice associated with sepsis/shock (167 $\mu \mathrm{mol} / \mathrm{l})$. Peak bilirubin levels were similar and in the middle range in the other groups; malignancy $251 \mu \mathrm{mol} / 1$, cirrhosis $224 \mu \mathrm{mol} / 1$, and hepatitis $250 \mu \mathrm{mol} / 1$ (see fig 2). When bilirubin was correlated with aspartate aminotransferase (AST), differences were found between the various groups. AST:bilirubin ratios were as follows: drug injury 16 , sepsis/shock 4.2 , hepatitis 1.1 , cirrhosis 1.0 , gall stone related 0.7 , and malignant obstruction 0.5 . Thus absolute bilirubin levels and bilirubin:AST ratios had some diagnostic value.

The study also examined the accuracy of the presumed diagnosis; 23 patients $(19 \%)$ were either given the wrong diagnosis or had no diagnosis cited. This was most common in the sepsis/shock group with 10 incorrect diagnoses, including viral hepatitis, carcinoma of the pancreas, and CBD stones. Nine patients with hepatocellular jaundice were also misdiagnosed; most notably four cases of drug, alcohol, or autoimmune related liver disease were incorrectly labelled viral hepatitis.

The postal questionnaire of gastroenterologists' and GPs' perceptions of what are the common causes of jaundice gave the following results (table 2). Gastroenterologists considered malignant biliary obstruction (2.0), gall stones (1.6), and alcoholic liver disease (1.2) to
Table 2 Commonest causes of marked jaundice, as perceived by gastroenterologists and general practitioners (GPS)

\begin{tabular}{lll}
\hline Diagnosis & $\begin{array}{l}\text { Cited by } \\
\text { consultants }\end{array}$ & Cited by GPs \\
\hline Malignant obstruction & 2.0 & 1.7 \\
Gall stones & 1.6 & 1.8 \\
Viral hepatitis & 0.5 & 1.6 \\
Alcoholic cirrhosis & 1.2 & 0.4 \\
Drug toxicity & 0.3 & 0.1 \\
Metastases & 0.1 & 0.3 \\
Other & 0.2 & 0.2 \\
\hline
\end{tabular}

be the commonest causes followed by viral hepatitis (0.5) and drug toxicity (0.3). Sepsis/ shock/postoperative causes were barely mentioned $(<0.1)$. GPs viewed gall stones (1.8), malignant biliary obstruction (1.7), and viral hepatitis (1.6) as the most important causes, followed by cirrhosis (0.4) and liver metastases (0.3).

\section{Discussion}

Our prospective study in a defined population revealed clinically obvious jaundice in 121 patients over a seven month period. This gives an annual incidence of 56 per 100000 assuming that all jaundiced patients had blood tests sent to the laboratory. It is likely that some jaundiced patients did not have blood tests because it was considered clinically unjustifiable - for example, those with terminal malignancy. We acknowledge that our survey will have excluded such patients. These values indicate that although jaundice is quite common in hospital practice it is rare in primary care. On average a GP with 2000 patients on his list will see only one patient with jaundice of this degree every year or two. Jaundice of a mild degree is much commoner but less likely to be noticed. Bilirubin levels below $50 \mu \mathrm{mol} / 1$ are not clinically detectable ${ }^{2}$ while bilirubin levels in the range $50-120 \mu \mathrm{mol} / 1$ should be detectable but may be missed because of skin pigmentation, poor artificial lighting, or simply failure of observation. We know from our laboratory records that during the survey period, 2221 patients had bilirubin levels elevated within this intermediate range of 26-120 $\mu \mathrm{mol} / 1$, the great majority being less than $50 \mu \mathrm{mol} / 1$. We decided against a detailed analysis of these patients because of uncertainties in knowing what proportion of those with a mildly elevated bilirubin will have escaped clinical detection and therefore blood sampling. We realise that had our arbitrarily chosen cut off point for bilirubin been 100 or 150 $\mu \mathrm{mol} / \mathrm{l}$, we would have seen a slightly different result but we do not feel that would have altered the central message of our study.

We are not aware of any published surveys of jaundice within a community directly comparable with ours. Several series are available from the 1960s and 1970s reflecting liver unit and hospital practice of the time, ${ }^{3-5}$ but case ascertainment was variable and diagnoses were made without the benefits of hepatitis serology and modern imaging techniques.

We have shown quite distinct patterns for the causes of jaundice first detected in primary 
care compared with jaundice arising in hospital. In the primary care setting jaundice was most commonly due to biliary obstruction or to decompensated alcoholic liver disease. GPs often correctly categorised the type of jaundice from the clinical circumstances although they greatly overestimated the likelihood of viral hepatitis as a cause. Despite correctly diagnosing obstructive jaundice from biliary malignancy or gall stones, GPs often referred their patients inappropriately to surgical departments. In hospitals serving this community, biliary investigations and therapeutic intervention are provided solely by physicians and radiologists rather than surgeons, and surgery is rarely required. The time honoured convention of referring jaundiced patients from primary care to surgical services simply delays effective management.

Jaundice appearing de novo in hospitalised patients has a very different diagnostic profile; sepsis and hepatic hypoxia were frequently responsible, particularly in the ITU, surgical, and oncology wards. The possibility of septic and hypoxic damage to the liver was not well recognised by the attending clinicians who often supposed viral hepatitis or drug toxicity to be the cause. In fact there were no cases of hospital acquired viral hepatitis during the study period. It is always difficult to exclude drug toxicity with complete certainty in postoperative patients and those in the ITU with multiorgan failure as they have often received a wide range of medications. ${ }^{6}$ We cannot rule out a contributory effect from drugs such as anaesthetic agents, analgesics, diuretics, inotropes, and antibiotics but no drug which is generally accepted as hepatotoxic was identified in this group. In support of our observations a recent study from North East England showed that over $50 \%$ of reported hepatic drug reactions were erroneous and that the actual cause of liver dysfunction was sepsis/ shock in more than one third of those misattributions.?

Elevated bilirubin is often seen in septic and shocked patients in the absence of primary liver or biliary disease ${ }^{8-12}$; several authors ${ }^{1011}$ have shown high mortality rates in septic patients who develop jaundice, but minor elevations of bilirubin in sepsis are very common and have little prognostic significance. ${ }^{13}$ The aetiology of hyperbilirubinaemia in sepsis is multifactorial; it is generally cholestatic ${ }^{14}$ and it has been postulated that it may be caused by circulating endotoxins ${ }^{15}$ although hepatic ischaemia and haemolysis may also play a role. ${ }^{6}{ }^{12}$

The diagnostic groupings used in our study were not always mutually exclusive. Some patients with gall stones had cholangitis and so it was difficult to separate them from the sepsis group but for our analysis we classified them as gall stone related. There were also two patients with underlying alcoholic cirrhosis who had sepsis causing temporary decompensation of their liver disease; these were included under cirrhosis. Patients with sepsis were often hypotensive and hypoxic and it was not always clear which was the dominant process in causing the increase in bilirubin, thus we considered it best to combine sepsis/shock into one group.

Although sepsis and hypoxia are known to cause jaundice, this is given little prominence in major textbooks of liver disease $\mathrm{e}^{16}$ or general medicine. ${ }^{17}$ It is perhaps for this reason that attending clinicians may not include sepsis/ shock in the differential diagnosis of jaundice. We have made similar observations in patients with marked elevations in aspartate transaminase ${ }^{18}$; again the cause was often sepsis/shock and this was frequently missed in hospital practice.

The number of patients with marked jaundice caused by gall stones was much lower than we expected. During the period of our study 61 patients had common bile duct stones at ERCP yet only 16 were picked up by our biochemical survey. In the remaining 45 cases the bilirubin level was below our cut off value of $120 \mu \mathrm{mol} / 1$. Thus our study indicates that obstructive jaundice with a bilirubin level greater than $120 \mu \mathrm{mol} / 1$ is much more likely to be caused by malignant obstruction than common bile duct stones.

We have previously shown that alcohol rather than viral hepatitis is the main cause of primary liver disease in South Wales ${ }^{19}$ so it was not surprising to find in our current study that alcoholic liver disease was the commonest cause of hepatocellular jaundice. This is quite different from published experience in the USA $^{20}$ and Canada ${ }^{21}$ where viral hepatitis dominates clinical hepatology. In our community acute viral hepatitis is extremely uncommon; only two cases from a population of 369000 over a seven month period. On statistical grounds a presumptive clinical diagnosis of viral hepatitis is unlikely to prove correct in South Wales.

Our survey of the perceptions on jaundice held by senior British gastroenterologists and hepatologists are revealing. It is possible that the pattern of jaundice is quite different in South Wales from the rest of the UK but we suspect that there are more similarities than differences. There is only a very small immigrant population in West Glamorgan, which would predict lower levels of chronic viral hepatitis than in some regions of Britain. There is no regional liver unit in South Wales so we might expect differences between our experience and that of colleagues in liver units who deal with many tertiary referrals. In particular, liver unit hepatologists will see disproportionate numbers of jaundiced patients suitable for transplantation or with acute liver failure and so will perceive primary cholestatic diseases, hepatitis C, and drug induced liver injury as common causes of jaundice.

We were surprised by the perceptions of local GPs who view viral hepatitis as an important cause of jaundice in the community, whereas this is not the case. Acute hepatitis A and B are exceptionally rare in South Wales and hepatitis C, either icteric or anicteric, is uncommon. During the study period our local Public Health Laboratory serving a population of 980000 in South and West Wales performed 6231 hepatitis serology tests and identified 
only one case of hepatitis A, one of acute hepatitis B, 19 chronic carriers of hepatitis B, and 38 cases of hepatitis C, 33 of whom were anicteric and five mildly icteric (personal communication Dr M Isaac and Dr A Lewis).

Gastroenterologists put drug jaundice quite high on their list of causes but in our survey it was not very common. The relative infrequency of hepatic adverse drug reactions was emphasised by a recent report from North East England which identified only 10-15 cases each year from that region. ${ }^{7}$

In conclusion, we have put some perspective on what causes obvious jaundice in this part of South Wales and we have highlighted the differences between these observations and the perceptions of consultant gastroenterologists and primary care physicians. We believe the findings may be more generally applicable to other parts of the UK with similar populations.

JGCK is grateful to the many BSG members and local GPs who not only responded to the questionnaire but often gave additional pertinent observations about jaundice, and particularly thanks Professor J Neuberger for his constructive comments on the paper.

1 Pearlman FC, Lee RTY. Detection and measurement of total bilirubin in serum with surfactant as solubilizing agents. Clin Chem 1974;20:447-53.

2 Brown AN, Sheiner LB, Cohen SN. Evaluation of bilirubin in a liver screening panel. $7 A M A$ 1992;268:1542.

3 Bjorneboe $M$, Tuersen $O$, Olsen S. Infective hepatitis and toxic jaundice in a municipal hospital during a five year period: Incidence and prognosis. Acta Med Scand 1967; 182:491-501.
4 Stern RB, Knill-Jones RP, Williams R. Use of computer program for diagnosing jaundice in district hospitals and program for diagnosing jaundice in district

5 Malchow-Møller A, Matzen P, Bjerregaard B, et al. Causes Malchow-Møller A, Matzen P, Bjerregaard B, et al. Causes
and characteristics of 500 consecutive cases of jaundice. and characteristics of 500 consec
Scand $\mathcal{F}$ Gastroenterol 1981;16:1-6.

6 Crawford JM, Boyer JL. Clinicopathology conferences: inflammation induced cholestasis. Hepatology 1998;28: 253-60

7 Aithal GP, Rawlins MD, Day CP. Accuracy of hepatic adverse drug reaction reporting in one English health region. BMF 1999;319:1541.

8 Schmid M, Hefti ML, Gattiker R, et al. Benign postoperative intrahepatic cholestasis. N Engl F Med 1965;272:545-50.

9 Ledingham IMcA, McArdle CS. Prospective study of the treatment of septic shock. Lancet 1978;I: 1194-7.

10 Banks JG, Foulis AK, Ledingham IMcA, et al. Liver function in septic shock. $\mathcal{F}$ Clin Pathol 1982;35:1249-52.

11 Franson TR, Hierholzer WJ, LaBrecque DR. Frequency Franson TR, Hierholzer W, LaBrecque DR. Frequency bacteremia. Rev Infect Dis 1985;7:1-9.

12 Moseley RM. Sepsis and cholestasis. Clin Liver Dis 1999;3: $465-75$

13 Sikula E, Guetta V, Keynan A, et al. Abnormalities in bilirubin and liver enzyme levels in adult patients with bacteremia. Arch Intern Med 1989;149:2246-8.

14 Zimmerman HJ, Fang M, Utili R, et al. Jaundice due to bacterial infection. Gastroenterology 1979;77:362-74.

15 Utili R, Abernethy CO, Zimmerman HJ. Inhibition of $\mathrm{Na}^{+}$ $\mathrm{K}^{+}$-ATPase by endotoxin: a possible mechanism for endotoxin-induced cholestasis. F Infect Dis 1977;136:583-7

16 Sherlock S, Dooley J. Diseases of the liver and biliary system. Oxford: Blackwell Scientific Publications, 1996.

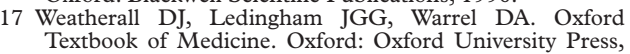
Textbook.

18 Whitehead MW, Hawkes ND, Hainsworth I, et al. A prospective study of the causes of notably raised aspartate transaminase of liver origin. Gut 1999;45:129-33.

19 Kingham JGC. Clinical hepatology: alcoholic liver disease not viral hepatitis predominates in South Wales. Hepatology 1997;25:1297.

20 Bach N. The significance of alcoholic liver disease to contemporary clinical hepatology. Hepatology 1996;24: 959-60.

21 Byron D, Minuk GY. Clinical hepatology: profile of an urban hospital based practice. Hepatology 1996;24:813-15. 